\title{
Preparation and Diastereoselective ortho-Metalation of Chiral Ferrocenyl Imidazolines: Remarkable Influence of LDA as Metalation Additive
}

René Peters* and Daniel F. Fischer

Laboratorium für Organische Chemie, ETH Zürich, Hönggerberg HCI E111,Wolfgang-Pauli-Str. 10, CH-8093 Zürich, Switzerland

peters@org.chem.ethz.ch 


\section{Table of Contents}

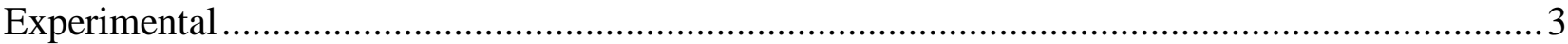

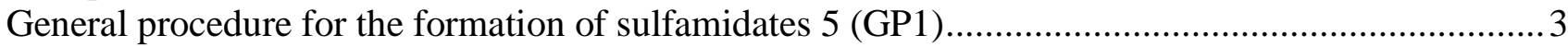

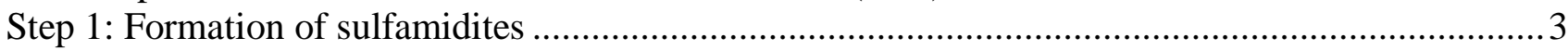

(4S,5R)-3,4-Dimethyl-5-phenyl-[1,2,3] oxathiazolidine 2-oxide …............................................

(4S,5S)-3,4-Dimethyl-5-phenyl-[1,2,3] oxathiazolidine 2-oxide .................................................

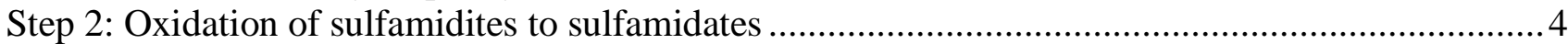

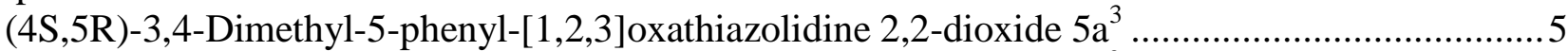

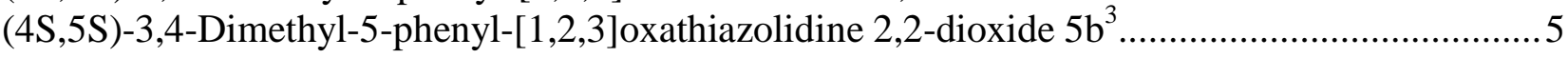

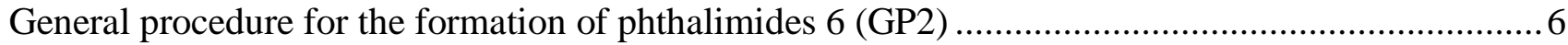

(1'S,2'S)-2-(2-Methylamino-1-phenyl-propyl)-isoindole-1,3-dione 6a.......................................... 6

(1'R,2'S)-2-(2-Methylamino-1-phenyl-propyl)-isoindole-1,3-dione 6b ........................................

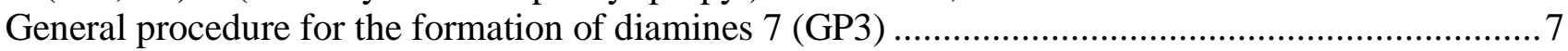

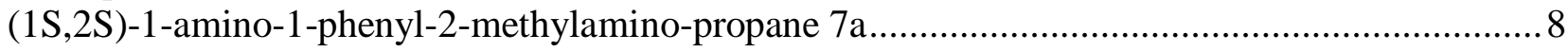

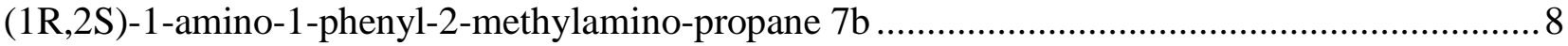

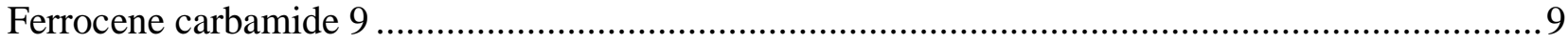

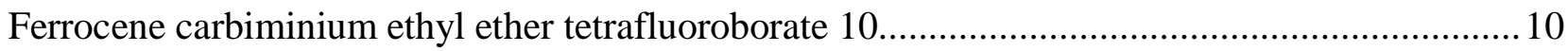

General procedure for the formation of ferrocenyl imidazolines 11 / 12 (GP4) ................................ 10

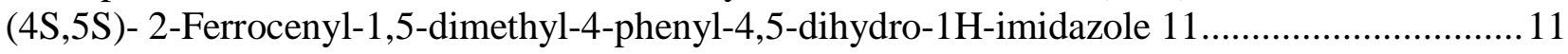

(4R,5S)-2-Ferrocenyl-1,5-dimethyl-4-phenyl-4,5-dihydro-1H-imidazole 12 ............................... 11

General procedure for the ortho-functionalization of ferrocenyl imidazolines 11 / 12 with $\mathrm{t}-\mathrm{BuLi}$ /

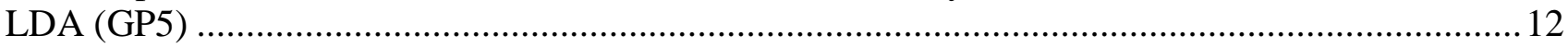

$\mathrm{S}_{\mathrm{p}}$-(4S,5S)-1,5-Dimethyl-2-(2-methyl-ferrocenyl)-4-phenyl-4,5-dihydro-1H-imidazole 13bA..... 13

$\mathrm{R}_{\mathrm{p}}$-(4S,5S)-1,5-Dimethyl-4-phenyl-2-(2-diphenylhydroxymethyl-ferrocenyl)-4,5-dihydro-1H-

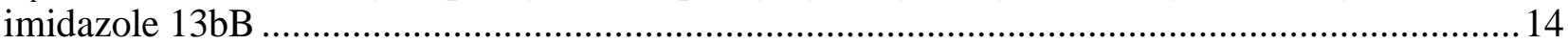

$\mathrm{R}_{\mathrm{p}}$-(4S,5S)-1,5-Dimethyl-4-phenyl-2-(2-diphenylphosphanyl-ferrocenyl)-4,5-dihydro-1H-

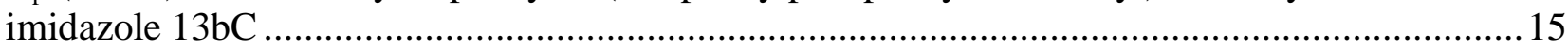

$\mathrm{R}_{\mathrm{p}}$-(4S,5S)-1,5-Dimethyl-4-phenyl-2-(2-p-tolylsulfanyl-ferrocenyl)-4,5-dihydro-1H-imidazole

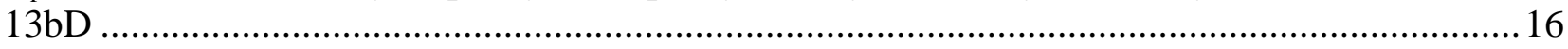

$\mathrm{R}_{\mathrm{p}}$-(4R,5S)-1,5-Dimethyl-2-(2-methyl-ferrocenyl)-4-phenyl-4,5-dihydro-1H-imidazole 14bA .... 17 $\mathrm{S}_{\mathrm{p}}$-(4R,5S)-1,5-Dimethyl-4-phenyl-2-(2-diphenylhydroxymethyl-ferrocenyl)-4,5-dihydro-1Himidazole $14 \mathrm{bB}$

General procedure for the ortho-functionalization of ferrocenyl imidazolines $11 / 12$ with t-BuLi

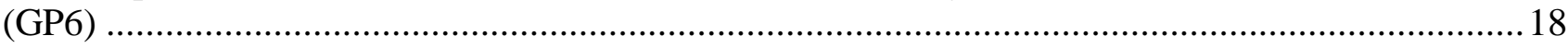

$\mathrm{R}_{\mathrm{p}}$-(4S,5S)-1,5-Dimethyl-2-(2-methyl-ferrocenyl)-4-phenyl-4,5-dihydro-1H-imidazole 13aA..... 18 $\mathrm{S}_{\mathrm{p}}$-(4S,5S)-1,5-Dimethyl-4-phenyl-2-(2-diphenylhydroxymethyl-ferrocenyl)-4,5-dihydro-1Himidazole $13 \mathrm{aB}$

Screening of conditions for the diastereoselective ortho metalation of ferrocenyl imidazoline $11 \ldots .21$ 


\section{Experimental}

All reactions were carried out in oven dried glassware $\left(150{ }^{\circ} \mathrm{C}\right)$ under an atmosphere of nitrogen. Chemicals were purchased from ABCR, Acros, Aldrich or Fluka and used as is unless mentioned otherwise. Evaporation of organic solutions was achieved by rotary evaporation with a water bath temperature below $45{ }^{\circ} \mathrm{C}$. Product purification by flash column chromatography was accomplished using silica gel $60(32-63 \mu \mathrm{m}$ particle size from Fluka) at 0.1 to 0.6 bar pressure. Technical grade solvents were used for chromatography and distilled prior to use. Thin-layer chromatography (TLC) was performed on Merck silica gel $60 \mathrm{~F}_{254}$ glass plates. Melting points were measured using a Büchi 535 melting point apparatus in open glass capillaries and are uncorrected. NMR spectra were recorded at room temperature on a Varian Mercury operating at $300 \mathrm{MHz}\left({ }^{1} \mathrm{H}\right), 75 \mathrm{MHz}\left({ }^{13} \mathrm{C}\right)$ and $122 \mathrm{MHz}$ $\left({ }^{31} \mathrm{P}\right)$ respectively. Residual solvent signals are internally referenced. Chemical shift $\delta$ is referred in terms of ppm, coupling constants $J$ are given in Hz. Following abbreviations classify the multiplicity: $\mathrm{s}$ $=$ singlet, $\mathrm{d}=$ doublet, $\mathrm{t}=$ triplet, $\mathrm{q}=$ quartett, $\mathrm{m}=$ multiplet or unresolved, $\mathrm{br}=$ broad signal. Infrared spectra were recorded on a Perkin-Elmer spektrum RX I FT-IR System and reported in $\mathrm{cm}^{-1}$. Samples were prepared in thin film technique. Combustion analysis was performed by the Mikroelementaranalytisches Laboratorium at ETH Zurich. Mass spectra were obtained from the ETH Zurich MS Service. ESI-mass spectra were recorded using a TSQ 7000 and HI-RES MALDI using an Ion Spec Ultima HR FT-ICR MS MALDI-FT-ICR MS employing the DHB-tl (2,5-dihydroxy-benzoic acid-two layers) method at 4.7 Tesla. High resolution EI mass spectra were performed on a Micromass AutoSpec Ultima and were calibrated with perfluorotributylamine (PFTBA) prior to data acquisition.

\section{General procedure for the formation of sulfamidates 5 (GP1)}

\section{Step 1: Formation of sulfamidites ${ }^{1}$}

To an ice-cooled solution of the corresponding aminoalcohol and imidazole (4.0 equiv) in DCM (2.3 $\mathrm{mL} / \mathrm{mmol}$ ) were successively added a solution of thionyl chloride (1.5 equiv) in DCM (1.0 mL / mmol) and triethylamine (2.0 equiv). After $1.5 \mathrm{~h}$ at $0{ }^{\circ} \mathrm{C}$, the reaction was quenched by addition of water $(2.3 \mathrm{~mL} / \mathrm{mmol})$. The organic phase was washed with the same amount of water, then dried over $\mathrm{MgSO}_{4}$. Removal of the solvent gave the corresponding crude sulfamidites as mixtures of two diastereomers, which were directly used in the next step without further purification.

\footnotetext{
${ }^{1}$ Modification of the following literature procedure: Williams, A. J.; Chakthong, S.; Gray, D.; Lawrence, R. M.; Gallagher, T. Org. Let., 2003, 5, 811 .
} 
(4S,5R)-3,4-Dimethyl-5-phenyl-[1,2,3]oxathiazolidine 2-oxide ${ }^{2}$

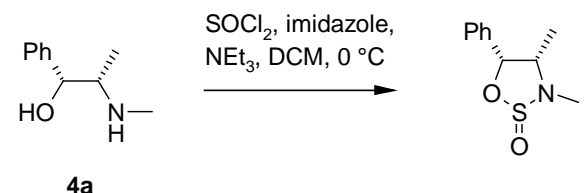

$(1 R, 2 S)$-ephedrine $(14.30 \mathrm{~g}, 86.5 \mathrm{mmol})$ and imidazole $(23.60 \mathrm{~g}, 4.0$ equiv) were treated according to GP1 (step 1) with thionylchloride (15.47g, 1.5 equiv) and triethylamine (17.50g, 2.0 equiv) to yield 18.20 g crude sulfamidite ( $d r \sim 60: 40$, yield: $100 \%$ by weight) as colorless oil, which solidifies after some hours at room temperature.

${ }^{1} \mathrm{H}$ NMR (300 MHz, $\left.\mathbf{C D C l}_{3}, 2{ }^{\circ} \mathbf{C}\right)$ ) major isomer $\delta=7.41-7.21(\mathrm{~m}, 5 \mathrm{H}$, arom. $H), 5.90(\mathrm{~d}, 1 \mathrm{H}, J=$ 6.9, $\mathrm{OCH}$ ), $3.95(\mathrm{~m}, 1 \mathrm{H}, \mathrm{NCH}), 2.66\left(\mathrm{~s}, 3 \mathrm{H}, \mathrm{NCH}_{3}\right), 0.79$ (d, 3H, $\left.J=6.9, \mathrm{CHCH}_{3}\right)$.

Minor isomer $\delta=7.21-7.41(\mathrm{~m}, 5 \mathrm{H}$, arom. $H), 5.64(\mathrm{~d}, 1 \mathrm{H}, J=6.9, \mathrm{OCH}), 3.77(\mathrm{~m}, 1 \mathrm{H}, \mathrm{NCH}), 2.85$ (s, $\left.3 \mathrm{H}, \mathrm{NCH}_{3}\right), 0.98\left(\mathrm{~d}, 3 \mathrm{H}, J=6.9, \mathrm{CHCH}_{3}\right)$.

\section{(4S,5S)-3,4-Dimethyl-5-phenyl-[1,2,3]oxathiazolidine 2-oxide ${ }^{3}$}

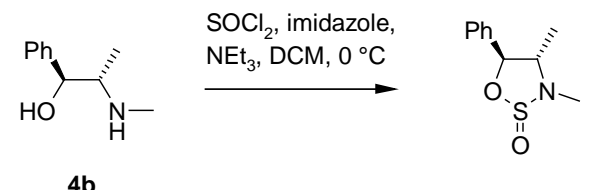

(1S,2S)-pseudoephedrine (10.00 g, $60.61 \mathrm{mmol})$ and imidazole $(16.32 \mathrm{~g}, 4.0$ equiv) were treated according to GP1 (step 1) with thionylchloride (9.24 g, 1.5 equiv) and triethylamine (12.12 g, 2.0 equiv) to yield $12.70 \mathrm{~g}$ crude sulfamidite ( $\mathrm{dr} \sim 1: 1$, yield: $99 \%$ by weight) as colorless solid.

${ }^{1} \mathrm{H}$ NMR $\left(300 \mathrm{MHz}, \mathbf{C D C l} 3,2{ }^{\circ} \mathbf{C}\right): \delta=7.47-7.35$ (m, 5H, arom. $H$ ), $4.94 \& 5.53(\mathrm{~d}, 1 \mathrm{H}, J=6,9$, $\mathrm{OCH}$ ), $3.24 \& 3.41(\mathrm{dq}, J=5.9, J=6.2,1 \mathrm{H}, \mathrm{NCH}), 2.62 \& 2.88\left(\mathrm{~s}, 3 \mathrm{H}, \mathrm{NCH}_{3}\right), 1.21 \& 1.27(\mathrm{~d}, J=6.2$, $\left.3 \mathrm{H}, \mathrm{CHCH}_{3}\right)$.

\section{Step 2: Oxidation of sulfamidites to sulfamidates}

Sodium periodate (1.5 equiv) and ruthenium (III) chloride trihydrate $(0.1 \mathrm{~mol} \%)$ were added to a solution of the crude sulfamidate in a 1:1 mixture of water and acetonitrile $(4.6 \mathrm{~mL} / \mathrm{mmol})$ at $0{ }^{\circ} \mathrm{C}$ and

\footnotetext{
${ }^{2}$ Known compound: Wudl, F.; Lee, T. B. K. J. Am. Chem. Soc. 1973, 95, 6349.

${ }^{3}$ Known compound: Van Dort, M. E.; Jung, Y.-W.; Sherman, P. S.; Kilbourn, M. R.; Wieland, D. M. J. Med. Chem. 1995, 38,810 .
} 
the resulting slurry was stirred for 2-4 h. After complete conversion, water $(2 \mathrm{~mL} / \mathrm{mmol})$ was added and the aqueous phase was extracted twice with MTBE ( $2 \mathrm{~mL} / \mathrm{mmol})$. The organic phase was washed with dilute aqueous sodium pyrrosulphite solution and then dried over $\mathrm{MgSO}_{4}$. The crude product was recrystallized from ethyl acetate or hexane.

\section{(4S,5R)-3,4-Dimethyl-5-phenyl-[1,2,3]oxathiazolidine 2,2-dioxide $5 \mathrm{a}^{3}$}

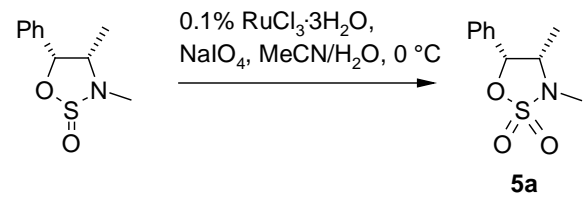

According to GP1 (step 2), treatment of $18.00 \mathrm{~g}$ crude (1R,2S)-3,4-dimethyl-5-phenyl[1,2,3] oxathiazolidine 2-oxide with $27.60 \mathrm{~g}$ sodium periodate and $20.0 \mathrm{mg}$ ruthenium (III) chloride trihydrate gave sulfamidate 5a (15.70 g, 80\% over two steps) after recrystallisation from EtOAc as a colorless solid $\left(d r>99: 1,{ }^{1} \mathrm{H}-\mathrm{NMR}\right)$.

$[\alpha]_{\mathrm{D}}^{25}\left(\mathrm{c}=1.00 \mathrm{~g} / \mathrm{dL}, \mathrm{CHCl}_{3}\right)=+23.0$.

Mp: $146.4{ }^{\circ} \mathrm{C}$.

${ }^{1}$ H NMR (300 MHz, CDCl3, $\left.21{ }^{\circ} \mathbf{C}\right): \delta=7.40(\mathrm{~m}, 5 \mathrm{H}$, arom. $H), 5.69(\mathrm{~d}, J=6.6,1 \mathrm{H}, \mathrm{OCH}), 3.89(\mathrm{dq}$, $J=6.0, J=9.3,1 \mathrm{H}, \mathrm{NCH}), 2.78\left(\mathrm{~s}, 3 \mathrm{H}, \mathrm{NCH}_{3}\right), 0.88\left(\mathrm{~d}, J=6.6,3 \mathrm{H}, \mathrm{CHCH}_{3}\right)$.

${ }^{13}$ C NMR (75 MHz, CDCl3, $\left.21{ }^{\circ} \mathbf{C}\right): \delta=133.2,129.3,128.6,127.0,84.7,59.6,30.8,14.0$.

IR (film): $v=1496,1461,1386,1325,1281,1202,1167,1119,1079,1036$.

MS (EI): $m / z 146.1\left[100,\left(\mathrm{M}-\mathrm{SO}_{3} \mathrm{H}\right)^{+}\right], 163.1\left[20,\left({\mathrm{M}-\mathrm{SO}_{2}}^{+}\right]\right.$.

Anal. Calcd for $\mathbf{C}_{10} \mathbf{H}_{13} \mathbf{N O}_{3} S:$ C, 52.85; H, 5.76 ; N, 6.16 . Found: C, 52.92 ; H, 5.81; N, 6.14.

(4S,5S)-3,4-Dimethyl-5-phenyl-[1,2,3] oxathiazolidine 2,2-dioxide $5 b^{3}$

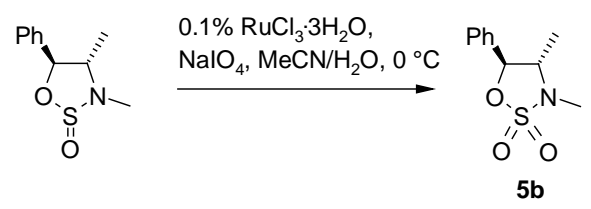

According to GP1 (step 2), treatment of $13.00 \mathrm{~g}$ crude (1S,2S)-3,4-dimethyl-5-phenyl$[1,2,3]$ oxathiazolidine 2 -oxide with sodium periodate $(15.00 \mathrm{~g})$ and ruthenium (III) chloride trihydrate 
(15.0 $\mathrm{mg})$ gave sulfamidate $\mathbf{5 b}(12.79 \mathrm{~g}, 94 \%$ over two steps) after recrystallisation from hexane as a colorless solid $\left(d r>99: 1,{ }^{1} \mathrm{H}-\mathrm{NMR}\right)$.

Mp: $66.5^{\circ} \mathrm{C}$.

$[\alpha]_{\mathrm{D}}^{25}\left(\mathrm{c}=1.30 \mathrm{~g} / \mathrm{dL}, \mathrm{CHCl}_{3}\right)=+23.8$.

${ }^{1}$ H NMR (300 MHz, CDCl3, $\left.21{ }^{\circ} \mathbf{C}\right): \delta=7.44(\mathrm{~m}, 5 \mathrm{H}$, arom. $H), 5.17(\mathrm{~d}, J=9.3,1 \mathrm{H}, \mathrm{OCH}), 3.45(\mathrm{~m}$, $1 \mathrm{H}, \mathrm{NCH}), 2.79\left(\mathrm{~s}, 3 \mathrm{H}, \mathrm{NCH}_{3}\right), 1.26\left(\mathrm{~d}, J=6.3,3 \mathrm{H}, \mathrm{CHCH}_{3}\right)$.

${ }^{13}$ C NMR (75 MHz, CDCl3, $\left.2{ }^{\circ} \mathrm{C}\right): 133.5,130.0,128.9,127.2,88.2,63.4,30.9,14.3$.

IR (film): $v=1498,1456,1382,1342,1276,1207,1176$.

MS (EI): $m / z 146.1\left[100,\left(\mathrm{M}-\mathrm{SO}_{3} \mathrm{H}\right)^{+}\right]$.

Anal. Calcd for $\mathbf{C}_{10} \mathrm{H}_{13} \mathrm{NO}_{3} \mathrm{~S}: \mathrm{C}, 52.85 ; \mathrm{H}, 5.76$; N, 6.16. Found: C, 53.02 ; H, 5.70; N, 6.04.

\section{General procedure for the formation of phthalimides 6 (GP2)}

A stirred suspension of sulfamidate 5 and potassium phthalimide (1.10-1.20 equiv) in abs. DMF (2.7 to $3.4 \mathrm{~mL} / \mathrm{mmol}$ ) was heated to $90-100{ }^{\circ} \mathrm{C}$ resulting in the formation of a clear solution. After $2-3.5 \mathrm{~h}$, the mixture was cooled to $0{ }^{\circ} \mathrm{C}$ and aqueous $5 \mathrm{~N} \mathrm{HCl}$ (1.00 equiv) was added dropwise. After $60 \mathrm{~min}$, the mixture was allowed to warm to room temperature and stirring was continued for additional $2.5 \mathrm{~h}$. The mixture was then poured into cold aqueous $0.5 \mathrm{~N} \mathrm{Na}_{2} \mathrm{CO}_{3}\left(0{ }^{\circ} \mathrm{C}\right)$ and the aqueous phase was extracted with DCM. The organic phase was washed three times with brine and three times with water. After addition of 20 vol.\% of EtOH, the organic phase was evaporated to dryness. 6 was used further as a crude product.

\section{(1’S,2'S)-2-(2-Methylamino-1-phenyl-propyl)-isoindole-1,3-dione 6a}

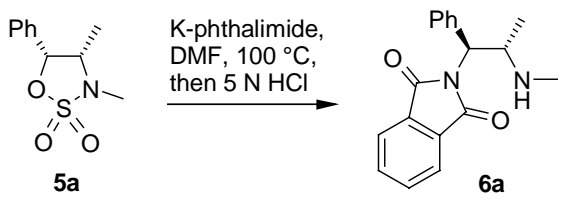

According to GP2, 5a $(5.605 \mathrm{~g}, 24.66 \mathrm{mmol})$ was treated with potassium phthalimide $(5.025 \mathrm{~g}, 1.10$ equiv) in $84 \mathrm{~mL} \mathrm{DMF}$ at $100{ }^{\circ} \mathrm{C}$ for $3.5 \mathrm{~h}$ furnishing $6 \mathbf{a}$ as a colorless solid $(6.819 \mathrm{~g}$, yield: $94 \%$ by weight, $\left.d r>99: 1,{ }^{1} \mathrm{H}-\mathrm{NMR}\right)$.

Mp: $119.2-119.9{ }^{\circ} \mathrm{C}$.

$[\alpha]_{\mathrm{D}}{ }^{25}\left(\mathrm{c}=2.40 \mathrm{~g} / \mathrm{dL}, \mathrm{CHCl}_{3}\right)=+16.2$. 
${ }^{1}$ H NMR (300 MHz, CDCl3, $\left.21{ }^{\circ} \mathbf{C}\right): \delta=7.78(\mathrm{~m}, 2 \mathrm{H}$, arom. $H), 7.66(\mathrm{dd}, J=7.3, J=4.0,2 \mathrm{H}$, arom. $H$ ), 7.60 (br. d, $J=6.9,2 \mathrm{H}$, arom. $H$ ), 7.28 (m, 3H, arom. $H$ ), $5.02(\mathrm{~d}, J=10.9,1 \mathrm{H}, \mathrm{PhCH}), 4.12(\mathrm{dq}$, $J=10.9, J=6.5,1 \mathrm{H}, \mathrm{MeCH}), 2.04\left(\mathrm{~s}, 3 \mathrm{H}, \mathrm{NCH}_{3}\right), 1.01\left(\mathrm{~d}, J=6.2,3 \mathrm{H}, \mathrm{CHCH}_{3}\right)$.

${ }^{13} \mathrm{C}$ NMR (75 MHz, CDCl3, $\left.21{ }^{\circ} \mathbf{C}\right): \delta=168.5,138.5,133.7,131.8,129.1,128.6,127.9,123.1,61.2$, 52.9, 32.6, 17.5 .

IR (film): $v=1708,1388,1329,1106$.

MS (MALDI): $m / z, 295\left[100, \mathrm{MH}^{+}\right]$.

Anal. Calcd for $\mathbf{C}_{18} \mathbf{H}_{18} \mathbf{N}_{2} \mathbf{O}_{2}$ : C, 73.45; H, 6.16; N, 9.52. Found: C, 73.16; H, 6.19; N, 9.39.

\section{(1'R,2'S)-2-(2-Methylamino-1-phenyl-propyl)-isoindole-1,3-dione 6b}

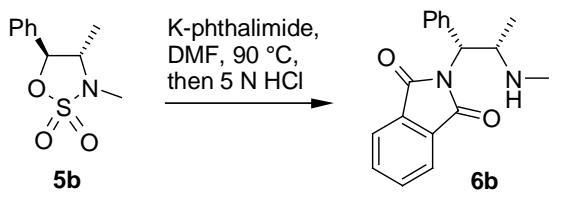

According to GP2, crude $5 \mathbf{b}(12.79 \mathrm{~g}$, ca. $60.0 \mathrm{mmol})$ was treated with potassium phthalimide $(11.90 \mathrm{~g}$, 1.18 equiv) in $150 \mathrm{ml} \mathrm{DMF}$ at $90{ }^{\circ} \mathrm{C}$ for $2 \mathrm{~h}$ furnishing $6 \mathbf{b}$ as a slightly yellow semisolid $(10.58 \mathrm{~g}, 60 \%$ by weight, $\left.d r>99: 1,{ }^{1} \mathrm{H}-\mathrm{NMR}\right)$, which was used in the next step without further purification. An analytical sample was prepared by column chromatography (MTBE $+3 \% \mathrm{NEt}_{3}$ ).

$[\alpha]_{\mathbf{D}}^{25}\left(\mathrm{c}=0.50 \mathrm{~g} / \mathrm{dL}, \mathrm{CHCl}_{3}\right)=-33.6$.

${ }^{1}$ H NMR (300 MHz, CDCl3, $\left.2{ }^{\circ} \mathbf{C}\right): \delta=7.75-7.34(\mathrm{~m}, 2 \mathrm{H}$, arom. $H), 7.62-7.59$ (m, 4H, arom. $\left.H\right)$, $7.34-7.25$ (m, 3H, arom. $H$ ), 5.07 (d, $J=10.8,1 \mathrm{H}, \mathrm{PhCH}), 4.10(\mathrm{dq}, J=6.3, J=6.0,1 \mathrm{H}, \mathrm{MeCH})$, $2.34\left(\mathrm{~s}, 3 \mathrm{H}, \mathrm{NCH}_{3}\right), 1.11$ (d, $\left.J=6.6,3 \mathrm{H}, \mathrm{CHCH}_{3}\right)$.

${ }^{13}$ C NMR (75 MHz, CDCl3, $\left.21{ }^{\circ} \mathbf{C}\right): \delta=168.0,137.7,133.7,131.6,129.2,128.7,128.2,123.1,60.2$, $53.0,33.1,16.5$.

IR (film): $v=2971,1765,1711,1466,1384,1330,1219$.

MS (MALDI): $m / z, 295.1\left[100 \%, \mathrm{MH}^{+}\right]$.

\section{General procedure for the formation of diamines 7 (GP3)}

To a stirred suspension of phthalimide 6 in ethanol $(3 \mathrm{~mL} / \mathrm{mmol})$ was added hydrazine monohydrate $(1.05-1.50$ equiv) at room temperature and the mixture was Subsequently heated to reflux for $1-4.5$ h. After cooling to room temperature, the mixture was filtrated and the filter cake was washed with EtOH. The filtrate was evaporated to dryness and the product was extracted from the residue with 
DCM. The procedure was the repeated with diisopropylether and the product was finally purified by Kugelrohr distillation.

\section{(1S,2S)-1-amino-1-phenyl-2-methylamino-propane $7 a^{4}$}

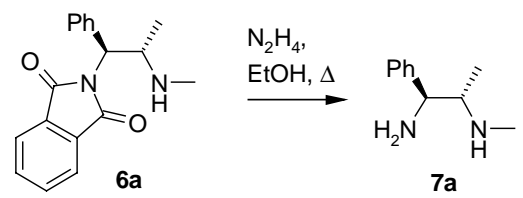

According to GP3, a solution of $6 \mathbf{a}(6.819 \mathrm{~g}, 23.17 \mathrm{mmol})$ in EtOH was treated with hydrazine monohydrate (1.18 mL, 1.05 equiv). Kugelrohr distillation (100 to $110{ }^{\circ} \mathrm{C}, 10^{-3}$ mbar) of the crude material gave rise to 7a as a colorless liquid $(2.818$ g, yield: $74 \%$, yield over 2 steps from 5a: 70\%, $d r>$ $\left.99: 1,{ }^{1} \mathrm{H}-\mathrm{NMR}\right)$.

$[\alpha]_{\mathrm{D}}^{25}\left(\mathrm{c}=2.80 \mathrm{~g} / \mathrm{dL}, \mathrm{CHCl}_{3}\right)=+55.5$.

${ }^{1}$ H NMR (300 MHz, CDCl3, $\left.21{ }^{\circ} \mathbf{C}\right): \delta=7.35$ - $7.21(\mathrm{~m}, 5 \mathrm{H}$, arom. $H), 3.61(\mathrm{~d}, J=7.8,1 \mathrm{H}, \mathrm{PhCH})$, $2.63(\mathrm{dq}, J=7.8, J=6.5,1 \mathrm{H}, \mathrm{MeCH}), 2.43$ (s, 3H, $\mathrm{NCH}_{3}$ ), 1.60 (br. s, 3H, NH$H_{2}$ and $\left.\mathrm{NHMe}\right), 0.87$ (d, $\left.J=6.5,3 \mathrm{H}, \mathrm{CHCH}_{3}\right)$.

${ }^{13} \mathrm{C}$ NMR (75 MHz, CDCl3, $\left.21^{\circ} \mathbf{C}\right): \delta=144.6,128.3,126.9,61.3,60.8,34.0,16.6$.

IR (film): $v=3027,2968,2794,1600,1453,1374,1091$.

MS (ESI): $m / z 175.3\left[100 \%, \mathrm{M}_{2} \mathrm{H}^{+}-2 \mathrm{C}_{6} \mathrm{H}_{5}\right], 165.3\left[20 \%, \mathrm{MH}^{+}\right]$.

Anal. Calcd for $\mathbf{C}_{10} \mathbf{H}_{16} \mathbf{N}_{\mathbf{2}}$ : C, 73.13; H, 9.82; N, 17.06. Found: C, 72.85; H, 9.59; N, 17.06 .

\section{$(1 R, 2 S)-1$-amino-1-phenyl-2-methylamino-propane $7 b^{5}$}

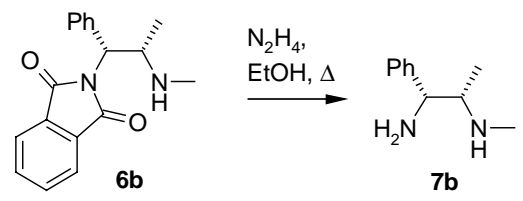

According to GP3, a solution of crude $6 \mathbf{b}(10.58 \mathrm{~g}$, ca. $35.0 \mathrm{mmol})$ in EtOH was treated with hydrazine monohydrate $\left(2.90 \mathrm{~mL}, 1.5\right.$ equiv). Kugelrohr distillation $\left(100{ }^{\circ} \mathrm{C}, 10^{-3}\right.$ mbar $)$ of the crude material gave rise to $\mathbf{7 b}$ as a colorless liquid (3.225 g, yield over 2 steps from $\mathbf{5 b}$ : 49\%, $\left.d r>99: 1,{ }^{1} \mathrm{H}-\mathrm{NMR}\right)$.

\footnotetext{
${ }^{4}$ Known compound: Tytgat, D.; Gelbcke, M. New J. Chem. 1991, 15, 293.

${ }^{5}$ Known compound: Tytgat, D.; Gelbcke, M.; Smith, D. F. Pharmazie 1990, 45, 835.
} 
$[\alpha]_{\mathrm{D}}^{25}\left(\mathrm{c}=2.0 \mathrm{~g} / \mathrm{dL}, \mathrm{CHCl}_{3}\right)=+0.2$.

${ }^{1}$ H NMR (300 MHz, CDCl3, $\left.21{ }^{\circ} \mathbf{C}\right): \delta=7.30-7.19(\mathrm{~m}, 5 \mathrm{H}$, arom. $H), 3.91(\mathrm{~d}, J=5.1,1 \mathrm{H}, \mathrm{PhCH})$, $2.70(\mathrm{dq}, J=5.1, J=1.4,1 \mathrm{H}, \mathrm{MeCH}), 2.37$ (s, 3H, NCH $), 1.40$ (br. s, 3H, NHMe and $\left.\mathrm{NH}_{2}\right), 0.94$ (d, $\left.J=6.3,3 \mathrm{H}, \mathrm{CHCH}_{3}\right)$.

${ }^{13} \mathbf{C}$ NMR (75 MHz, CDCl3, $\left.21^{\circ} \mathbf{C}\right): \delta=143.6,128.2,126.9,60.7,58.8,34.2,14.9$.

IR (film): $v=3304,3058,3026,2969,2793,1601,1451,1324$.

MS (ESI): $m / z 175.3\left[100 \%, \mathrm{M}_{2} \mathrm{H}^{+}-2 \mathrm{C}_{6} \mathrm{H}_{5}\right], 165.3\left[15 \%, \mathrm{MH}^{+}\right]$.

Anal. Calcd for $\mathbf{C}_{\mathbf{1 0}} \mathbf{H}_{\mathbf{1 6}} \mathbf{N}_{\mathbf{2}}$ : C, 73.13; H, 9.82; N, 17.06. Found: C, 72.92; H, 9.95; N, 16.90 .

\section{Ferrocene carbamide $9^{6}$}

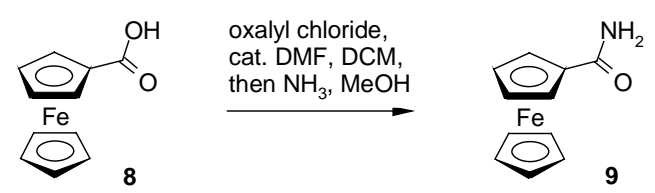

To a slurry of ferrocene carboxylic acid 8 (7.46 g, $32.4 \mathrm{mmol})$ in DCM (150 mL) were successively added dropwise at room temperature oxalyl chloride $(6.10 \mathrm{~g}, 48.0 \mathrm{mmol}, 1.5$ equiv) and a solution of DMF (1.0 mol/L, $1.0 \mathrm{mmol}, 0.03$ equiv) in DCM. After $45 \mathrm{~min}$ at room temperature, all volatiles were removed under reduced pressure. The dark red oily residue was dissolved in DCM (150 mL) and a solution of ammonia in methanol ( $7 \mathrm{~N}, 60 \mathrm{~mL}, 420 \mathrm{mmol}, 13$ equiv) was added within 5 min while cooling the reaction mixture with a water bath $\left(20{ }^{\circ} \mathrm{C}\right)$. The resulting slurry was stirred for $3 \mathrm{~h}$, then quenched by addition of water $(200 \mathrm{~mL})$. Extraction with MTBE (2 times $300 \mathrm{~mL}$ ) and drying over $\mathrm{MgSO}_{4}$ gave after removal of the solvent $(6.60 \mathrm{~g}, 90 \%)$ as bright orange solid.

Mp: $168^{\circ} \mathrm{C}\left(\mathrm{Lit}^{2}: 168^{\circ}-170{ }^{\circ} \mathrm{C}\right)$.

${ }^{1}$ H NMR (300 MHz, DMSO-d6, $\left.21{ }^{\circ} \mathbf{C}\right): \delta=7.30 \& 6.90$ (br., $1 \mathrm{H}$ each, $\left.\mathrm{NH} H_{2}\right), 4.75(\mathrm{dt}, J=3.8, J=1.9$, $\left.2 \mathrm{H}, \mathrm{o}_{-} \mathrm{C}_{5} \mathrm{H}_{4}\right), 4.32\left(\mathrm{dt}, J=3.8, J=1.9,2 \mathrm{H}, \mathrm{m}-\mathrm{C}_{5} \mathrm{H}_{4}\right), 4.16\left(\mathrm{~s}, 5 \mathrm{H}, \mathrm{C}_{5} \mathrm{H}_{5}\right)$.

${ }^{13}$ C NMR (75 MHz, DMSO-d6, $\left.21^{\circ} \mathbf{C}\right): \delta=170.8,76.2,69.8,69.2,68.2$.

MS (EI): $m / z$ 229.0, $\left[100 \%, \mathbf{M}^{+}\right]$.

Anal. Calcd for $\mathbf{C}_{11} \mathbf{H}_{11}$ NOFe: C, 57.68; H, 4.84; N, 6.11. Found C, 57.95; H, 4.90; N, 6.09.

\footnotetext{
${ }^{6}$ Modification of the procedure reported by Arimoto, F. S.; Haven Jr., A. C. J. Am. Chem. Soc. 1955, 77, 6295.
} 
Ferrocene carbiminium ethyl ether tetrafluoroborate 10

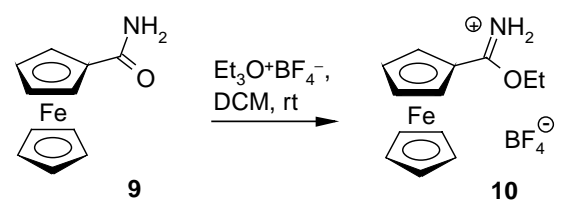

To ferrocene carbamide $9(2.997 \mathrm{~g}, 13.0 \mathrm{mmol})$ was added at room temperature a solution of triethyloxonium tetrafluoroborate $(2.558 \mathrm{~g}, 13.5 \mathrm{mmol}, 1.04$ equiv.) in $80 \mathrm{ml}$ DCM. The solution was stirred for $5 \mathrm{~h}$ and used for imidazoline formation without further purification.

${ }^{1}$ H NMR (300 MHz, CDCl3, $\left.21^{\circ} \mathbf{C}\right): \delta=9.04 \& 8.56\left(\mathrm{~s}, 1 \mathrm{H}\right.$ each, $\left.\mathrm{NH}_{2}{ }^{+}\right), 5.08\left(\mathrm{t}, J=1.6,2 \mathrm{H}, \mathrm{o}_{-} \mathrm{C}_{5} H_{4}\right)$, $4.73\left(\mathrm{t}, J=1.6, \mathrm{~m}_{-} \mathrm{C}_{5} \mathrm{H}_{4}\right), 4.54\left(\mathrm{q}, \mathrm{J}=7.1,2 \mathrm{H}, \mathrm{CH}_{2} \mathrm{Me}\right), 4.35\left(\mathrm{~s}, 5 \mathrm{H}, \mathrm{C}_{5} \mathrm{H}_{5}\right), 1.55\left(\mathrm{t}, J=6.8,3 \mathrm{H}, \mathrm{CH}_{3}\right)$.

${ }^{13} \mathrm{C}$ NMR (75 MHz, $\left.\mathbf{C D C l}_{3}, 21^{\circ} \mathbf{C}\right): \delta=179.3,74.7,71.2,69.8,69.0,64.6,13.6$.

MS (ESI): $m / z 258.1\left[100, \mathbf{M}^{+}-\mathrm{BF}_{4}^{-}\right]$.

IR: 3364, 3215, 3115, 1683, 1565, 1515, 1412, 1390, 1072.

Anal. Calcd for $\mathbf{C}_{13} \mathbf{H}_{16} \mathbf{N O B F}_{4}$ Fe: C, 45.27; H, 4.68; N, 4.06. Found: C, 45.33; H, 4.60; N, 3.98.

\section{General procedure for the formation of ferrocenyl imidazolines 11 / 12 (GP4)}

To a stirred solution of diamine 7 in abs. DCM or DCE $(10 \mathrm{~mL} / \mathrm{mmol})$ was slowly added at $0{ }^{\circ} \mathrm{C}$ within $30 \mathrm{~min}$ a solution of the iminoether salt in the same solvent $(4.5 \mathrm{~mL} / \mathrm{mmol})$ while vigorously stirring the resulting yellow-orange solution. After $30 \mathrm{~min}$, the mixture was allowed to warm to room temperature and stirring was continued for $16 \mathrm{~h}$. Subsequently, $90 \%$ of solvent were removed by evaporation and diisopropylether $(13 \mathrm{~mL} / \mathrm{mmol})$ was added resulting in the precipitation of an orange solid. The mixture was kept at $-22{ }^{\circ} \mathrm{C}$ for $48 \mathrm{~h}$ and the supernatant was removed by decantation and the residue was washed with diisopropylether $(2.2 \mathrm{~mL} / \mathrm{mmol})$. The oily residue was dissolved in DCM and washed twice with $1 \mathrm{~N} \mathrm{NaOH}$. The organic phase was dried over $\mathrm{Na}_{2} \mathrm{CO}_{3}$ or $\mathrm{CaH}_{2}$ and evaporated to dryness after filtration. 
(4S,5S)- 2-Ferrocenyl-1,5-dimethyl-4-phenyl-4,5-dihydro-1H-imidazole 11

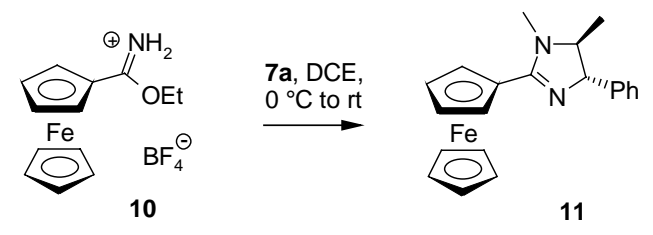

According to GP4, a solution of 7a $(798.3 \mathrm{mg}, 1.08$ equiv) in DCE was treated with 10 (1552.1 mg, $4.50 \mathrm{mmol})$ giving the crude product $(1608.0 \mathrm{mg})$, which was recrystallized from $\mathrm{Et}_{2} \mathrm{O} /$ hexane furnishing 11 as a yellow brown solid (1392.8 mg, yield: 86\%, $\left.d r>99: 1,{ }^{1} \mathrm{H}-\mathrm{NMR}\right)$.

$[\alpha]_{\mathrm{D}}^{25}\left(\mathrm{c}=0.135 \mathrm{~g} / \mathrm{dL}, \mathrm{CHCl}_{3}\right)=-58.4$.

Mp: $84.8-86.4^{\circ} \mathrm{C}$.

${ }^{1} \mathrm{H}$ NMR (300 MHz, CDCl3, $\left.21{ }^{\circ} \mathbf{C}\right): \delta=7.37-7.22(\mathrm{~m}, 5 \mathrm{H}$, arom. $H), 4.80\left(\mathrm{~m}, 1 \mathrm{H}, o-\mathrm{C}_{5} H_{4} \mathrm{R}\right), 4.72$ $\left(\mathrm{m}, 1 \mathrm{H}, o-\mathrm{C}_{5} H_{4} \mathrm{R}\right), 4.45(\mathrm{~d}, J=8.7,1 \mathrm{H}, \mathrm{PhCH}), 4.36\left(\mathrm{t}, J=1.9,2 \mathrm{H}, m-\mathrm{C}_{5} H_{4} \mathrm{R}\right), 4.25(\mathrm{~s}, 5 \mathrm{H}, \mathrm{Cp}), 3.28$ $(\mathrm{dq}, J=9.0, J=5.9,1 \mathrm{H}, \mathrm{MeCH}), 3.12\left(\mathrm{~s}, 3 \mathrm{H}, \mathrm{NCH}_{3}\right), 1.35\left(\mathrm{~d}, J=6.2,3 \mathrm{H}, \mathrm{CHCH}_{3}\right)$.

${ }^{13} \mathrm{C}$ NMR (75 MHz, CDCl3, $\left.21{ }^{\circ} \mathbf{C}\right): \delta=165.0,144.7,128.6,127.1,75.9,74.6,70.0,69.9,69.8,69.7$, 69.4, 33.9, 18.8 .

IR (film): $v=1574,1492,1451,1392,1309,1105,1033,1002$.

MS (ESI): $m / z 359.2\left[100 \%, \mathrm{MH}^{+}\right]$.

HRMS (MALDI) $m / e$ : Calcd for $\left(\mathrm{MH}^{+}\right) \mathbf{C}_{21} \mathbf{H}_{23} \mathbf{F e N}_{2}$ : 359.1205. Found: 359.1212.

Anal. Calcd for $\mathbf{C}_{21} \mathbf{H}_{22} \mathbf{F e N}_{2}$ : C, 70.40; H, 6.19; N, 7.82. Found: C, 70.28; H, 6.33; N, 7.82 .

(4R,5S)-2-Ferrocenyl-1,5-dimethyl-4-phenyl-4,5-dihydro-1H-imidazole 12
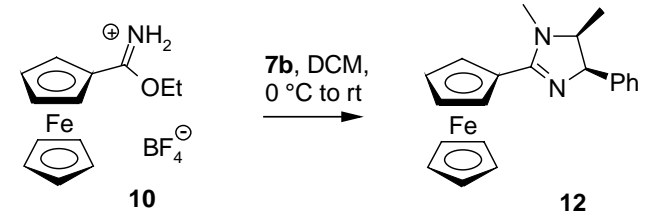

According to GP4, a solution of $\mathbf{7 b}(1207.0 \mathrm{mg}, 1.00$ equiv) in DCM was treated with $\mathbf{1 0}(2529.0 \mathrm{mg}$, $35.00 \mathrm{mmol}$ ) to give the crude product, which was dissolved in benzene and dried over $\mathrm{CaH}_{2}$ to yield $12\left(2.256 \mathrm{~g}, 85 \%, d r>99: 1,{ }^{1} \mathrm{H}-\mathrm{NMR}\right)$ as a dark red oil after evaporation of solvent. $\mathbf{1 0}$ was stored as a frozen solution in benzene at $-18{ }^{\circ} \mathrm{C}$. 
$[\alpha]_{\mathrm{D}}^{25}\left(\mathrm{c}=0.165 \mathrm{~g} / \mathrm{dL}, \mathrm{CHCl}_{3}\right)=+108.8$.

${ }^{1}$ H NMR (300 MHz, CDCl3, $\left.21^{\circ} \mathbf{C}\right): \delta=7.36-7.20(\mathrm{~m}, 5 \mathrm{H}$, arom. $H), 5.05(\mathrm{~d}, J=10.0,1 \mathrm{H}, \mathrm{CHPh})$, $4.76\left(\mathrm{~m}, 1 \mathrm{H}, o-\mathrm{C}_{5} \mathrm{H}_{4} \mathrm{R}\right), 4.72\left(\mathrm{~m}, 1 \mathrm{H}, o-\mathrm{C}_{5} \mathrm{H}_{4} \mathrm{R}\right), 4.37-4.35\left(\mathrm{~m}, 2 \mathrm{H}, m-\mathrm{C}_{5} H_{4} \mathrm{R}\right), 4.27$ (s, 5H, Cp), 3.80 $(\mathrm{dq}, J=10.3, J=6.5,1 \mathrm{H}, \mathrm{MeCH}), 3.05\left(\mathrm{~s}, 3 \mathrm{H}, \mathrm{NCH}_{3}\right), 0.80\left(\mathrm{~d}, J=6.6,3 \mathrm{H}, \mathrm{CHCH}_{3}\right)$.

${ }^{13} \mathrm{C}$ NMR (75 MHz, CDCl3, $\left.21{ }^{\circ} \mathbf{C}\right): \delta=166.0,139.7,128.0,127.8,126.7,71.3,70.1,69.7,69.5$, $68.8,64.1,33.4,14.5$.

IR (film): $v=.3084,3024,2967,2925,1587,1573,1492,1451,1393,1334,1288,1251,1208,1105$, 1082, 1030, 1001 .

MS (EI): $358.112\left[100 \%, \mathrm{M}^{+}\right]$.

HRMS (EI) $m / e$ : Calcd for $\left(\mathrm{M}^{+}\right) \mathbf{C}_{21} \mathbf{H}_{22} \mathrm{FeN}_{2}$ : 358.1127. Found: 358.1129.

Anal. Calcd for $\mathbf{C}_{21} \mathbf{H}_{22} \mathbf{F e N}_{2}$ : C, 70.40; H, 6.19; N, 7.82. Found: C, 70.74; H, 6.57; N, 7.48.

\section{General procedure for the ortho-functionalization of ferrocenyl imidazolines 11/ 12 with t-BuLi /} LDA (GP5)

A solution of 11 or 12 in abs. THF $(13 \mathrm{~mL} / \mathrm{mmol})$ was cooled to $-78{ }^{\circ} \mathrm{C}$ for $45 \mathrm{~min}$. Subsequently, LDA (1.00 or 1.50 equiv, $0.21 \mathrm{M}$ in THF, prepared from diisopropylamine and n-butyllithium at $0{ }^{\circ} \mathrm{C} 2$ $-48 \mathrm{~h}$ prior to use) was slowly added. After 30 to $45 \mathrm{~min}$ at $-78{ }^{\circ} \mathrm{C}, t-\mathrm{BuLi}(0.95$ or 1.00 equiv, $1.45 \mathrm{M}$ in pentane) was added dropwise. After the indicated time at $-78{ }^{\circ} \mathrm{C}$, the corresponding electrophile was added dropwise. The reaction was quenched after the specified time by addition of aqueous $\mathrm{NaOH}(0.1$ $\mathrm{M})$ and the product was extracted with either MTBE or $\mathrm{Et}_{2} \mathrm{O}$. The organic layer was filtrated over a plug of $\mathrm{Na}_{2} \mathrm{SO}_{4}$ before evaporation under reduced pressure. The crude material was purified by column chromatography and/or recrystalization allowing to recover unreacted imidazoline starting material in good yield. 


\section{$S_{p}$-(4S,5S)-1,5-Dimethyl-2-(2-methyl-ferrocenyl)-4-phenyl-4,5-dihydro-1H-imidazole 13bA}
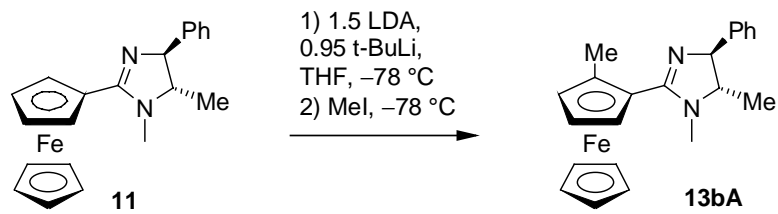

According to GP5, $11(57.5 \mathrm{mg}, 160.5 \mu \mathrm{mol})$ was treated with LDA (1.50 equiv) and $t$-BuLi (0.95 equiv). After $5.5 \mathrm{~h}$ at $-78{ }^{\circ} \mathrm{C}$, methyliodide $(52.5 \mu \mathrm{L}, 5.25$ equiv) was added dropwise. The reaction was quenched after $10 \mathrm{~min}$. The crude product (54.0 mg, $\left.d r=12: 1,{ }^{1} \mathrm{H}-\mathrm{NMR}\right)$ was purified by column chromatography (cyclohexane / EtOAc $\left.(8: 1), 3 \% \mathrm{NEt}_{3}\right)$ furnishing diastereomerically pure $(d r>99: 1$, $\left.{ }^{1} \mathrm{H}-\mathrm{NMR}\right)$ 13bA as orange crystals (39.6 mg, yield: $66 \%$ ).

$[\alpha]_{\mathrm{D}}^{25}\left(\mathrm{c}=0.91 \mathrm{~g} / \mathrm{dL}, \mathrm{CHCl}_{3}\right)=+260.6$.

Mp: $101.2-102.2{ }^{\circ} \mathrm{C}$.

${ }^{1}$ H NMR (300 MHz, CDCl3, $\left.21{ }^{\circ} \mathbf{C}\right): \delta=7.36(\mathrm{~d}, J=4.4,4 \mathrm{H}$, arom. $H), 7.27$ (m, 1H, arom. $\left.H\right), 4.61$ $(\mathrm{d}, J=9.9,1 \mathrm{H}, \mathrm{PhCH}), 4.33\left(\mathrm{dd}, 1 \mathrm{H}, J=2.5, J=1.4, \mathrm{C}_{5} H_{3} \mathrm{R}_{2}\right), 4.24\left(\mathrm{dd}, 1 \mathrm{H}, J=2.2, J=1.6, \mathrm{C}_{5} H_{3} \mathrm{R}_{2}\right)$, $4.20(\mathrm{~s}, 5 \mathrm{H}, \mathrm{Cp}), 4.15\left(\mathrm{t}, J=2.5,2 \mathrm{H}, m-\mathrm{C}_{5} H_{4} \mathrm{R}\right), 3.07(\mathrm{dq}, J=9.9, J=6.1,1 \mathrm{H}, \mathrm{MeCH}), 2.75(\mathrm{~s}, 3 \mathrm{H}$, $\left.\mathrm{NCH}_{3}\right), 2.26\left(\mathrm{~s}, 3 \mathrm{H}, \mathrm{CpCH}_{3}\right), 1.37\left(\mathrm{~d}, J=6.1,3 \mathrm{H}, \mathrm{CHCH}_{3}\right)$.

${ }^{13}$ C NMR (75 MHz, CDCl3, $\left.21{ }^{\circ} \mathbf{C}\right): \delta=165.3,144.7,128.3,126.83,126.76,85.7,76.2,70.5,70.1$, 69.4, 68.0, 66.4, 34.1, 18.3, 14.4 .

IR (film): $v=1601,1482,1451,1374,1332,1304,1271,1205,1105,1019$.

MS (ESI): $m / z, 373.1\left[100, \mathrm{MH}^{+}\right]$.

HRMS (MALDI) m/e: Calcd for $\left(\mathrm{MH}^{+}\right) \mathbf{C}_{22} \mathbf{H}_{25} \mathbf{F e N}$ : 373.1362. Found: 373.1368.

Anal. Calcd for $\mathbf{C}_{22} \mathbf{H}_{24} \mathbf{F e N}$ : C, 70.98; H, 6.50; N, 7.52. Found: C, 70.96; H, 6.48; N, 7.42. 


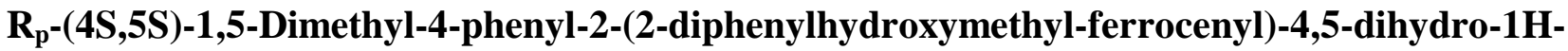
imidazole $13 \mathrm{bB}$

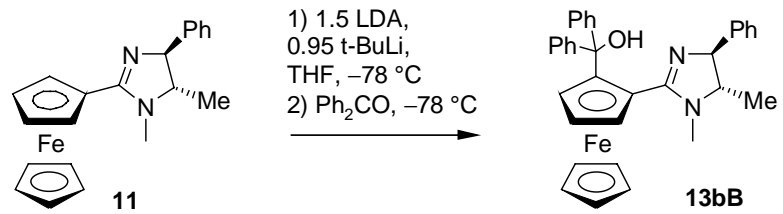

According to GP5, $11(109.0 \mathrm{mg}, 304.2 \mu \mathrm{mol})$ was treated with LDA (1.50 equiv) and $t$-BuLi (0.95 equiv). After $5.25 \mathrm{~h}$ at $-78{ }^{\circ} \mathrm{C}$, a solution of benzophenone in THF (1.00 equiv, $0.6 \mathrm{M}$ ) was added dropwise. The reaction was quenched after $10 \mathrm{~min}$. The crude product $\left(160.5 \mathrm{mg}, d r=12: 1,{ }^{1} \mathrm{H}\right.$ NMR) was purified by column chromatography (cyclohexane / EtOAc (8:1), 3\% $\mathrm{NEt}_{3}$ ) and Subsequently by recrystalization from $\mathrm{Et}_{2} \mathrm{O} /$ hexane (1:2) furnishing 13bB $\left(d r>99: 1,{ }^{1} \mathrm{H}-\mathrm{NMR}\right)$ as orange crystals $(82.8 \mathrm{mg}$, yield: $50 \%)$.

$[\alpha]_{\mathbf{D}}^{25}\left(\mathrm{c}=0.25 \mathrm{~g} / \mathrm{dL}, \mathrm{CHCl}_{3}\right)=+273.3$.

Mp: $218.6-219.1{ }^{\circ} \mathrm{C}$ (decomp.).

${ }^{1}$ H NMR (300 MHz, CDCl3, $\left.21^{\circ} \mathbf{C}\right): \delta=9.73$ (s, 1H, OH), 7.52 (br. d, $J=6.9,2 \mathrm{H}$, arom. $H$ ), $7.36-$ $7.12(\mathrm{~m}, 11 \mathrm{H}$, arom. $H), 6.63(\mathrm{~m}, 2 \mathrm{H}$, arom. $H), 4.58\left(\mathrm{dd}, 1 \mathrm{H}, J=2.4, J=1.4, \mathrm{C}_{5} H_{3} \mathrm{R}_{2}\right), 4.44(\mathrm{~d}, J=$ 11.0, 1H, $\mathrm{PhCH}), 4.36(\mathrm{~s}, 5 \mathrm{H}, \mathrm{Cp}), 4.21\left(\mathrm{t}, J=2.5,2 \mathrm{H}, m-\mathrm{C}_{5} H_{4} \mathrm{R}\right), 3.56(\mathrm{dd}, 1 \mathrm{H}, J=2.5, J=1.6$, $\left.\mathrm{C}_{5} H_{3} \mathrm{R}_{2}\right), 2.73\left(\mathrm{~s}, 3 \mathrm{H}, \mathrm{NCH}_{3}\right), 2.62(\mathrm{dq}, J=11.3, J=6.3,1 \mathrm{H}, \mathrm{MeCH}), 1.27\left(\mathrm{~d}, J=6.0,3 \mathrm{H}, \mathrm{CHCH}_{3}\right)$.

${ }^{13} \mathrm{C}$ NMR (75 MHz, CDCl3, $\left.21{ }^{\circ} \mathbf{C}\right): \delta=169.1,150.5,147.1,142.6,128.2,128.0,127.7,127.1,127.0$, $126.8,126.4,126.3,102.7,77.3,75.7,73.7,72.5,71.1,70.9,68.2,66.1,35.3,17.8$.

IR (film): $v=3057,2923,2852,1593,1452,1370,1214,1107,1049,1019$.

MS (ESI): $m / z, 541.1\left[15, \mathrm{MH}^{+}\right], 523.1\left[100, \mathrm{MH}^{+}-\mathrm{H}_{2} \mathrm{O}\right]$.

Anal. Calcd for $\mathbf{C}_{34} \mathbf{H}_{32} \mathrm{FeN}_{2} \mathrm{O}$ : C, 75.56; H, 5.97; N, 5.18. Found: C, 75.36; H, 6.16; N, 4.98. 


\section{$R_{p}$-(4S,5S)-1,5-Dimethyl-4-phenyl-2-(2-diphenylphosphanyl-ferrocenyl)-4,5-dihydro-1H- imidazole $13 \mathrm{bC}$}
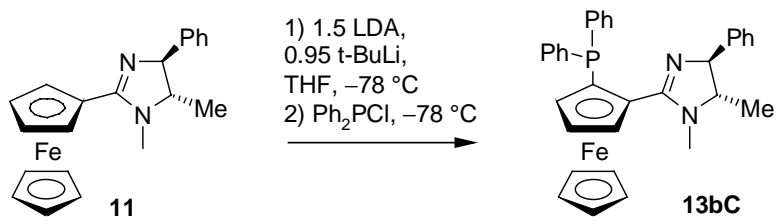

According to GP5, $11(54.5 \mathrm{mg}, 152.1 \mu \mathrm{mol})$ was treated with LDA (1.50 equiv) and $t$-BuLi $(0.95$ equiv). After $5 \mathrm{~h}$ at $-78{ }^{\circ} \mathrm{C}$, diphenylphospine chloride (1.10 equiv) was added dropwise. The reaction was quenched after $60 \mathrm{~min}$. The crude product (86.0 mg, $\left.d r=7: 1,{ }^{1} \mathrm{H}-\mathrm{NMR}\right)$ was purified by column chromatography (cyclohexane / EtOAc (8:1), 3\% $\mathrm{NEt}_{3}$ ) furnishing $\mathbf{1 3 b C}$ as a mixture of two diastereomers $\left(d r=8: 1,{ }^{1} \mathrm{H}-\mathrm{NMR}\right)$ as yellow oil (41.3 $\mathrm{mg}$, yield: $\left.50 \%\right)$ being very prone to oxidation. 13bC has to be stored at low temperature due to decomposition at room temperature.

$[\alpha]_{\mathrm{D}}^{25}\left(\mathrm{c}=0.15 \mathrm{~g} / \mathrm{dL}, \mathrm{C}_{6} \mathrm{H}_{6}\right)=+142.5$.

${ }^{1}$ H NMR (300 MHz, C6D6, $\left.21^{\circ} \mathbf{C}\right): \delta=7.68(\mathrm{~m}, 2 \mathrm{H}$, arom. $H), 7.43(\operatorname{td}, J=7.8, J=2.5,2 \mathrm{H}$, arom. $H)$, $7.31(\mathrm{~m}, 2 \mathrm{H}$, arom. H), $7.23(\mathrm{~m}, 2 \mathrm{H}$, arom. $H), 7.15-7.00(\mathrm{~m}, 7 \mathrm{H}, 2 \mathrm{H}$, arom. $H), 4.68(\mathrm{~d}, J=11.2,1 \mathrm{H}$, $\mathrm{PhCH}), 4.46\left(\mathrm{dt}, 1 \mathrm{H}, J=2.5, J=1.3, \mathrm{C}_{5} H_{3} \mathrm{R}_{2}\right), 4.27(\mathrm{~s}, 5 \mathrm{H}, \mathrm{Cp}), 4.06\left(\mathrm{t}, J=2.5,1 \mathrm{H}, \mathrm{C}_{5} H_{3} \mathrm{R}_{2}\right), 3.79(\mathrm{~m}$, $\left.1 \mathrm{HC}_{5} \mathrm{H}_{3} \mathrm{R}_{2}\right), 2.63(\mathrm{dq}, J=10.9, J=6.2,1 \mathrm{H}, \mathrm{MeCH}), 2.27$ (s, 3H, NCH$\left.H_{3}\right), 1.04$ (d, $\left.J=6.2,3 \mathrm{H}, \mathrm{CHCH}_{3}\right)$. ${ }^{13}$ C NMR (75 MHz, C6D6, $\left.21{ }^{\circ} \mathrm{C}\right): \delta=164.3,144.9,141.0(\mathrm{~d}, J=14.7), 139.7(\mathrm{~d}, J=16.5), 135.2(\mathrm{~d}$, $J=22.0), 133.3(\mathrm{~d}, J=19.6), 128.6,128.3,127.9,127.0,126.6,82.5(\mathrm{~d}, J=19.5), 80.6(\mathrm{~d}, J=14.7)$, $72.6(\mathrm{~d}, J=4.3), 71.6,71.2(\mathrm{~d}, J=6.7), 70.1,69.7,34.1,18.1$.

${ }^{31}$ P NMR (122 MHz, C6D6, $\left.21{ }^{\circ} \mathbf{C}\right): \delta=-19.9$ (minor diastereomer: -20.2).

IR (film): $v=3055,2963,1581,1538,1480,1451,1435,1349,1119,1050$.

MS (ESI): $m / z, 542.9\left[100, \mathrm{MH}^{+}\right]$.

HRMS (MALDI) $m / e$ : Calcd for $\mathbf{C}_{33} \mathbf{H}_{32} \mathbf{F e N} \mathbf{P}\left(\mathrm{MH}^{+}\right)$: 543.1647. Found: 543.1636.

Anal. Calcd for $\mathbf{C}_{33} \mathbf{H}_{31} \mathbf{F e N}_{2} \mathbf{P}$ : C, 73.07; H, 5.76; N, 5.16. Found: C, 72.99; H, 5.91; N, 4.69. 


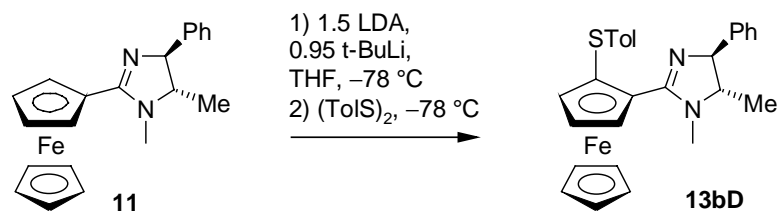

According to GP5, $11(52.8 \mathrm{mg}, 147.4 \mu \mathrm{mol})$ was treated with LDA (1.50 equiv) and $t$-BuLi $(0.95$ equiv). After $5.5 \mathrm{~h}$ at $-78{ }^{\circ} \mathrm{C}$, a solution of p-tolyldisulfide (1.10 equiv) in THF (1.0 M) was added dropwise. The reaction was quenched after $40 \mathrm{~min}$. The crude product $\left(80.1 \mathrm{mg}, d r=17: 1,{ }^{1} \mathrm{H}-\mathrm{NMR}\right)$ was purified by column chromatography (cyclohexane / EtOAc (8:1), 3\% $\mathrm{NEt}_{3}$ ) furnishing $\mathbf{1 3 b D}$ as a mixture of two diastereomers $\left(d r=10: 1,{ }^{1} \mathrm{H}-\mathrm{NMR}\right)$ as yellow oil (37.8 mg, yield: $\left.53 \%\right)$, which has to be stored at low temperature due to decomposition at room temperature.

$[\alpha]_{\mathrm{D}}^{25}\left(\mathrm{c}=1.05 \mathrm{~g} / \mathrm{dL}, \mathrm{CHCl}_{3}\right)=-202.1$.

${ }^{1}$ H NMR (300 MHz, C6D6, $\left.21^{\circ} \mathbf{C}\right): \delta=7.65(\mathrm{~d}, J=7.2,2 \mathrm{H}$, arom. $H), 7.32(\mathrm{t}, J=7.2,3 \mathrm{H}$, arom. $H)$, $7.26(\mathrm{~d}, J=8.1,2 \mathrm{H}$, arom. $H), 6.80(\mathrm{~d}, J=8.1,2 \mathrm{H}$, arom. $H), 4.77(\mathrm{~d}, J=10.6,1 \mathrm{H}, \mathrm{PhCH}), 4.46(\mathrm{dd}, J$ $\left.=2.5, J=1.3,1 \mathrm{H}, \mathrm{C}_{5} H_{3} \mathrm{R}_{2}\right), 4.40(\mathrm{~s}, 5 \mathrm{H}, \mathrm{Cp}), 4.35\left(\mathrm{dd}, J=2.5, J=1.3,1 \mathrm{H}, \mathrm{C}_{5} H_{3} \mathrm{R}_{2}\right), 4.02(\mathrm{t}, J=2.5$, $\left.1 \mathrm{H}, \mathrm{C}_{5} H_{3} \mathrm{R}_{2}\right), 2.78(\mathrm{dq}, J=10.6, J=6.2,1 \mathrm{H}, \mathrm{MeCH}), 2.12\left(\mathrm{~s}, 3 \mathrm{H}, \mathrm{CH}_{3}\right), 1.98\left(\mathrm{~s}, 3 \mathrm{H}, \mathrm{CH}_{3}\right), 1.04(\mathrm{~d}, J=$ $\left.6.2,3 \mathrm{H}, \mathrm{CHCH}_{3}\right)$.

${ }^{13}$ C NMR (75 MHz, C6D6, $\left.21{ }^{\circ} \mathbf{C}\right): \delta=163.3,145.3,137.1,134.9,129.5,128.7,128.4,126.9,82.7$, 81.0, 77.5, 75.3, 72.2, 70.1, 69.8, 68.9, 33.9, 20.8, 18.2.

IR (film): $v=2345,2341,1609,1570,1490,1451,1004$.

HRMS (MALDI) $m / e$ : Calcd for $\left(\mathrm{MH}^{+}\right) \mathbf{C}_{28} \mathbf{H}_{29} \mathbf{F e N} 2 \mathrm{~S}: 481.1394$. Found: 481.1385.

Anal. Calcd for $\mathbf{C}_{28} \mathrm{H}_{28} \mathrm{FeN}_{2} \mathrm{~S}$ : C, 70.00; H, 5.87; N, 5.83. Found: C, 69.81; H, 6.00; N, 5.99. 


\section{$R_{p}$-(4R,5S)-1,5-Dimethyl-2-(2-methyl-ferrocenyl)-4-phenyl-4,5-dihydro-1H-imidazole 14bA}
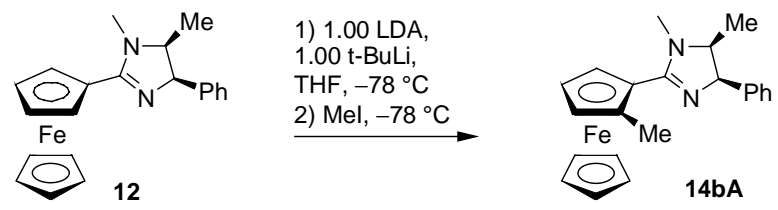

According to GP5, $12(100.0 \mathrm{mg}, 279.1 \mu \mathrm{mol})$ was treated with LDA (1.00 equiv) and $t$-BuLi (1.00 equiv). After $2.5 \mathrm{~h}$, MeI (52.0 mg, $366.1 \mu \mathrm{mol}, 1.30$ equiv.) was added dropwise. The reaction was quenched after $45 \mathrm{~min}$. Purification of the crude product $\left(d r=31: 1,{ }^{1} \mathrm{H}-\mathrm{NMR}\right)$ by column chromatography (cyclohexane / EtOAc (8:1), 3\% $\mathrm{NEt}_{3}$ ) and subsequivuent trituration with hexane gave 14bA as orange solid (43.0 mg, yield: $41 \%, d r=150: 1,{ }^{1} \mathrm{H}-\mathrm{NMR}$ )

Mp: $102.6-103.5^{\circ} \mathrm{C}$

$[\alpha]_{\mathbf{D}}^{25}\left(\mathrm{c}=0.70 \mathrm{~g} / \mathrm{dL}, \mathrm{CHCl}_{3}\right)=-95.6$.

${ }^{1} \mathrm{H}-\mathrm{NMR}\left(300 \mathrm{MHz}, \mathbf{C D C l}_{3}, \mathbf{2 1}^{\circ} \mathrm{C}\right): \delta=7.35-7.24(\mathrm{~m}, 5 \mathrm{H}$, arom. $H$ ), $5.20(\mathrm{~d}, J=10.2,1 \mathrm{H}, \mathrm{PhCH})$, $4.42\left(\mathrm{dd}, 1 \mathrm{H}, J=2.5, J=1.4, \mathrm{C}_{5} H_{3} \mathrm{R}_{2}\right), 4.22(\mathrm{~s}, 5 \mathrm{H}, \mathrm{Cp}), 4.15\left(\mathrm{t}, J=2.5,2 \mathrm{H}, m-\mathrm{C}_{5} H_{4} \mathrm{R}\right), 3.71(\mathrm{dq}, J=$ 10.6, $J=6.6,1 \mathrm{H}, \mathrm{MeCH}$ ), 2.68 (s, 3H, $\left.\mathrm{NCH}_{3}\right), 2.21\left(\mathrm{~s}, 3 \mathrm{H}, \mathrm{CpCH}_{3}\right), 0.77$ (d, $J=6.6,3 \mathrm{H}, \mathrm{CHCH}_{3}$ ).

${ }^{13} \mathbf{C}$ NMR (75 MHz, $\left.\mathbf{C D C l}_{3}, 2{ }^{\circ} \mathbf{C}\right): \delta=166.3,140.0,128.2,127.8,126.7,82.7,72.1,70.5,70.2,70.0$, $66.6,63.6,33.5,14.8,14.5$.

IR (film): $v$ = 2956, 2918, 1602, 1491, 1451, 1375, 1334, 1285, 1234, 1105, 1054.

MS (MALDI): $m / z 373.1\left[100, \mathrm{MH}^{+}\right]$.

Anal. Calcd for $\mathbf{C}_{22} \mathbf{H}_{24} \mathbf{F e N}_{2}$ : C, 70.98; H, 6.50; N, 7.52. Found: C, 70.95; H, 6.33; N, 7.38.

$S_{\mathrm{p}}$-(4R,5S)-1,5-Dimethyl-4-phenyl-2-(2-diphenylhydroxymethyl-ferrocenyl)-4,5-dihydro-1Himidazole 14bB

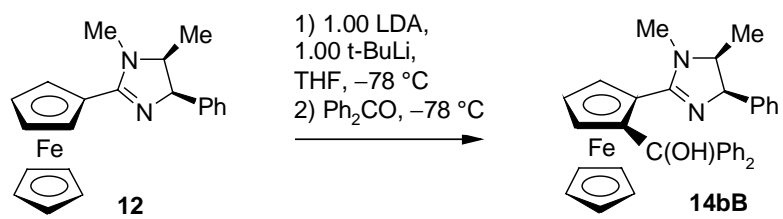

According to GP5, $12(100.0 \mathrm{mg}, 279.1 \mu \mathrm{mol})$ was treated with LDA (1.00 equiv) and $t$-BuLi (1.00 equiv). After $2.5 \mathrm{~h}$, a solution of benzophenone in THF (1.00 equiv, $0.6 \mathrm{M})$ was added dropwise. The reaction was quenched after 25 min. Purification of the crude product (conversion: 75\%, dr $=21: 1$, 
${ }^{1} \mathrm{H}-\mathrm{NMR}$ ) by column chromatography (cyclohexane / EtOAc (4:1), 3\% $\mathrm{NEt}_{3}$ ) and subsequent trituration with hexane gave $\mathbf{1 4 b B}$ as bright orange solid $\left(62.5 \mathrm{mg}, 41 \%, d r>99: 1,{ }^{1} \mathrm{H}-\mathrm{NMR}\right)$.

Mp: $185.5-186.5^{\circ} \mathrm{C}$ (decomp.).

$[\alpha]_{\mathrm{D}}^{25}\left(\mathrm{c}=1.52 \mathrm{~g} / \mathrm{dL}, \mathrm{CHCl}_{3}\right)=-258.0$.

${ }^{1}$ H NMR $\left(300\right.$ MHz, CDCl3, $\left.21{ }^{\circ} \mathbf{C}\right): \delta=9.97(\mathrm{~s}, 1 \mathrm{H}, \mathrm{OH}), 7.52(\mathrm{~m}, 2 \mathrm{H}$, arom. H), $7.35-7.04(\mathrm{~m}$, 13H, arom. $H$ ), $5.16(\mathrm{~d}, J=9.6,1 \mathrm{H}, \mathrm{PhCH}), 4.55\left(\mathrm{~m}, 1 \mathrm{H}, \mathrm{o}-\mathrm{C}_{5} H_{3} \mathrm{R}_{2}\right), 4.37(\mathrm{~s}, 5 \mathrm{H}, \mathrm{Cp}), 4.18(\mathrm{t}, J=2.5$, $\left.1 \mathrm{H}, \mathrm{o}_{-} \mathrm{C}_{5} \mathrm{H}_{3} \mathrm{R}\right), 3.80\left(\mathrm{dd}, 1 \mathrm{H}, J=6.5, J=9.7, \mathrm{CHCH}_{3}\right), 3.51\left(\mathrm{~m}, 1 \mathrm{H}, \mathrm{m}-\mathrm{C}_{5} \mathrm{H}_{3} \mathrm{R}_{2}\right), 2.78\left(\mathrm{~s}, 3 \mathrm{H}, \mathrm{NCH}_{3}\right)$, $0.18\left(\mathrm{~d}, J=6.3,3 \mathrm{H}, \mathrm{CHCH}_{3}\right)$.

${ }^{13}$ C NMR (75 MHz, CDCl3, $\left.21{ }^{\circ} \mathbf{C}\right): \delta=167.4,150.2,147.1,139.3,128.3,128.2,128.0,127.9,127.3$, $127.3,126.7,103.5,73.5,71.4,70.9,68.0,66.1,64.2,31.8,11.4$.

IR (film): $v=3057,1576,1451,1375,1255,1006$.

MS (EI): $m / z 540.2\left[100, \mathbf{M}^{+}\right]$.

Anal. Calcd for $\mathbf{C}_{34} \mathbf{H}_{32} \mathrm{FeN}_{2} \mathrm{O}$ : C, 75.56; H, 5.97; N, 5.18. Found: C, 75.22; H, 6.08; N, 5.05.

\section{General procedure for the ortho-functionalization of ferrocenyl imidazolines 11 / 12 with t-BuLi} (GP6)

A solution of 11 or 12 in THF $(13 \mathrm{~mL} / \mathrm{mmol})$ was cooled to $-78{ }^{\circ} \mathrm{C}$ for $20 \mathrm{~min}$. Subsequently, $t$-BuLi (1.00 to 1.10 equiv, $1.45 \mathrm{M}$ in pentane) was added dropwise. After the indicated time at $-78{ }^{\circ} \mathrm{C}$, the corresponding electrophile was added dropwise. The reaction was quenched after the specified time by addition of aqueous $\mathrm{NaOH}(0.1 \mathrm{M})$ and the product was extracted with either MTBE or $\mathrm{Et}_{2} \mathrm{O}$. The organic layer was filtrated over a plug of $\mathrm{Na}_{2} \mathrm{SO}_{4}$ prior to evaporation under reduced pressure. The crude material was purified by column chromatography and/or recrystalization.

\section{$R_{p}$-(4S,5S)-1,5-Dimethyl-2-(2-methyl-ferrocenyl)-4-phenyl-4,5-dihydro-1H-imidazole 13aA}
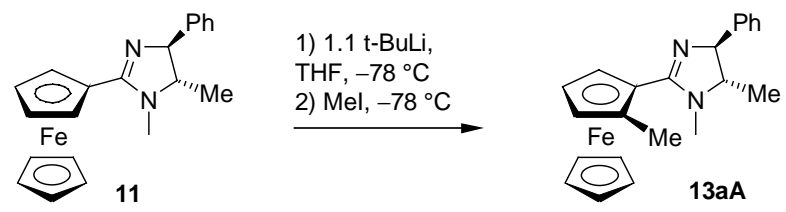

According to GP6, $11(11.0 \mathrm{mg}, 30.7 \mu \mathrm{mol})$ was treated with $t$-BuLi (1.10 equiv). After $2.5 \mathrm{~h}$ at -78 ${ }^{\circ} \mathrm{C}$, methyliodide $(2.9 \mu \mathrm{L}, 1.5$ equiv) was added. The reaction was quenched after $60 \mathrm{~min}$. The crude product $\left(11.0 \mathrm{mg}, d r=5: 1,{ }^{1} \mathrm{H}-\mathrm{NMR}\right)$ was purified by column chromatography (cyclohexane / EtOAc 
(8:1), 3\% $\left.\mathrm{NEt}_{3}\right)$ furnishing 13aA $\left(d r=20: 1,{ }^{1} \mathrm{H}-\mathrm{NMR}\right)$ as yellow-orange crystals (7.0 mg, yield: $61 \%)$.

$[\alpha]_{\mathrm{D}}^{25}\left(\mathrm{c}=0.39 \mathrm{~g} / \mathrm{dL}, \mathrm{CHCl}_{3}\right)=-84.3$.

Mp: $95.2-98.2^{\circ} \mathrm{C}$ (decomp.).

${ }^{1}$ H NMR (300 MHz, CDCl3, $\left.2{ }^{\circ} \mathbf{C}\right): \delta=7.41-7.24(\mathrm{~m}, 5 \mathrm{H}$, arom. $H), 4.53(\mathrm{~d}, J=8.4 \mathrm{~Hz}, 1 \mathrm{H}$, $\mathrm{PhCH}$ ), $4.35\left(\mathrm{dd}, 1 \mathrm{H}, J=2.5 \mathrm{~Hz}, J=1.6 \mathrm{~Hz}, \mathrm{C}_{5} H_{3} \mathrm{R}_{2}\right), 4.27\left(\mathrm{dd}, 1 \mathrm{H}, J=2.5 \mathrm{~Hz}, J=1.2 \mathrm{~Hz}, \mathrm{C}_{5} H_{3} \mathrm{R}_{2}\right)$, $4.20(\mathrm{~s}, 5 \mathrm{H}, \mathrm{Cp}), 4.19\left(\mathrm{~m}, 2 \mathrm{H}, m-\mathrm{C}_{5} H_{4} \mathrm{R}\right), 3.20(\mathrm{dq}, J=8.7 \mathrm{~Hz}, J=6.2 \mathrm{~Hz}, 1 \mathrm{H}, \mathrm{MeCH}), 2.97(\mathrm{~s}, 3 \mathrm{H}$, $\left.\mathrm{NCH}_{3}\right), 2.25\left(\mathrm{~s}, 3 \mathrm{H}, \mathrm{CpCH}_{3}\right), 1.35\left(\mathrm{~d}, J=6.5 \mathrm{~Hz}, 3 \mathrm{H}, \mathrm{CHCH}_{3}\right)$.

${ }^{13} \mathrm{C}$ NMR (75 MHz, CDCl3, $\left.2{ }^{\circ} \mathbf{C}\right): \delta=164.3,144.9,128.3,126.8,126.6,84.5,76.0,70.7,70.3,69.7$, 68.9, 67.1, 34.3, 18.1, 15.4 .

MS (MALDI): $m / z, 373.1\left[100, \mathrm{MH}^{+}\right]$.

IR (film): 2960, 2863, 1601, 1578, 1482, 1451, 1374, 1304, 1210, 1105, 1019, 1001.

Anal. Calcd for $\mathbf{C}_{22} \mathbf{H}_{24} \mathrm{FeN}_{2}$ : C, 70.98; H, 6.50; N, 7.52. Found: C, 70.88; H, 6.67; N, 7.37.

$S_{\mathrm{p}}$-(4S,5S)-1,5-Dimethyl-4-phenyl-2-(2-diphenylhydroxymethyl-ferrocenyl)-4,5-dihydro-1Himidazole $13 a B$
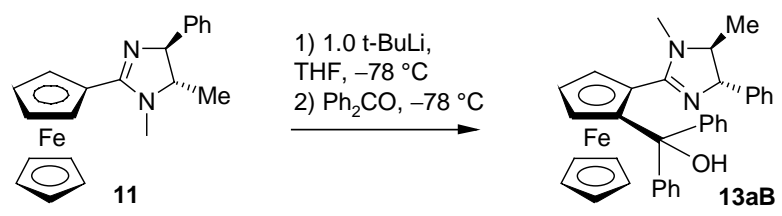

According to GP6, $11(50.0 \mathrm{mg}, 139.6 \mu \mathrm{mol})$ was treated with $t$-BuLi (1.00 equiv). After $4 \mathrm{~h}$ at $-78{ }^{\circ} \mathrm{C}$, a solution of benzophenone in THF (26.3 mg, 1.00 equiv, $0.6 \mathrm{M})$ was added dropwise. The reaction was quenched after $20 \mathrm{~min}$. The crude product $(77.5 \mathrm{mg}, \mathrm{dr}=3.7: 1)$ was purified by silica gel filtration (cyclohexane / EtOAc (8:1), 3\% $\mathrm{NEt}_{3}$ ) giving a mixture of diastereomers (46.1 mg, yield: $\left.59 \%, d r=4: 1,{ }^{1} \mathrm{H}-\mathrm{NMR}\right)$ and finally by trituration using $\mathrm{Et}_{2} \mathrm{O} /$ hexane furnishing $13 \mathrm{aB}$ (26.5 mg, yield: $\left.34 \%, d r=19: 1,{ }^{1} \mathrm{H}-\mathrm{NMR}\right)$ as yellow-orange crystals.

$[\alpha]_{\mathrm{D}}^{25}\left(\mathrm{c}=0.75 \mathrm{~g} / \mathrm{dL}, \mathrm{CHCl}_{3}\right)=+10.8$.

Mp: $173.5-174.5^{\circ} \mathrm{C}$ (decomp.).

${ }^{1}$ H NMR (300 MHz, CDCl3, $\left.21{ }^{\circ} \mathbf{C}\right): \delta=9.57$ (br. s, 1H, OH), $7.56(\mathrm{~d}, J=7.2 \mathrm{~Hz}, 2 \mathrm{H}$, arom. $H$ ), 7.38 $-7.10(\mathrm{~m}, 13 \mathrm{H}$, arom. $H), 4.46\left(\mathrm{dd}, 1 \mathrm{H}, J=2.5 \mathrm{~Hz}, J=1.2 \mathrm{~Hz}, \mathrm{C}_{5} H_{3} \mathrm{R}_{2}\right), 4.43(\mathrm{~s}, 5 \mathrm{H}, \mathrm{Cp}), 4.18(\mathrm{t}, J=$ 
$\left.2.5 \mathrm{~Hz}, 2 \mathrm{H}, m-\mathrm{C}_{5} H_{4} \mathrm{R}\right), 3.97(\mathrm{~d}, J=7.5 \mathrm{~Hz}, 1 \mathrm{H}, \mathrm{PhCH}), 3.56\left(\mathrm{dd}, 1 \mathrm{H}, J=2.5 \mathrm{~Hz}, J=1.5 \mathrm{~Hz}, \mathrm{C}_{5} H_{3} \mathrm{R}_{2}\right)$, $3.30(\mathrm{dq}, J=7.5 \mathrm{~Hz}, J=6.2 \mathrm{~Hz}, 1 \mathrm{H}, \mathrm{MeCH}), 2.65\left(\mathrm{~s}, 3 \mathrm{H}, \mathrm{NCH}_{3}\right), 0.59\left(\mathrm{~d}, J=6.2 \mathrm{~Hz}, 3 \mathrm{H}, \mathrm{CHCH}_{3}\right)$. ${ }^{13} \mathbf{C}$ NMR (75 MHz, CDCl3, $\left.21{ }^{\circ} \mathbf{C}\right): \delta=165.0,150.2,146.1,144.4,128.3,127.4,127.2,127.0,126.9$, $126.8,126.3,126.0,105.2,77.2,73.9,72.6,71.2,71.0,67.6,65.8,66.7,30.3,18.2$.

MS (MALDI): $m / z 541.2\left[100, \mathrm{MH}^{+}\right]$.

IR (film): 3058, 2853, 1591, 1451, 1272, 1218, 1107, 1048, 1018.

Anal. Calcd for $\mathbf{C}_{34} \mathbf{H}_{32} \mathbf{F e N}_{2} \mathbf{O}$ : C, 75.56; H, 5.97; N, 5.18. Found: C, 75.30; H, 5.98; N, 5.14. 


\section{Screening of conditions for the diastereoselective ortho metalation of ferrocenyl imidazoline 11}

\begin{tabular}{|c|c|c|c|c|c|c|c|c|}
\hline entry & solvent & equiv base & equiv additive & $\begin{array}{c}\mathrm{T} \\
{\left[{ }^{\circ} \mathrm{C}\right]}\end{array}$ & $\begin{array}{l}\text { precooling of } \mathbf{1 1} \\
\text { in THF prior to } \\
\text { addition of the } \\
\text { additive } \\
\text { [min] }\end{array}$ & $\begin{array}{l}\text { precooling of } \\
\mathbf{1 1}+\text { additive in } \\
\text { THF prior to } \\
\text { addition of the } \\
\text { base } \\
\text { [min] }\end{array}$ & $\begin{array}{c}\text { conversion } \\
{[\%]}\end{array}$ & $\begin{array}{c}d r \\
13 \mathbf{a A}: \mathbf{1 3 b A}\end{array}$ \\
\hline 1 & $\mathrm{Et}_{2} \mathrm{O}$ & $1.1 \mathrm{~s}-\mathrm{BuLi}$ & - & -78 & 5 & - & 60 & $1.5: 1$ \\
\hline 2 & $\mathrm{Et}_{2} \mathrm{O}$ & $1.1 \mathrm{n}-\mathrm{BuLi}$ & - & -78 & 10 & - & 55 & $2.1: 1$ \\
\hline 3 & $\mathrm{Et}_{2} \mathrm{O}$ & $1.1 \mathrm{t}-\mathrm{BuLi}$ & - & -78 & 10 & - & 54 & $3.0: 1$ \\
\hline 4 & Et2O & $1.1 \mathrm{PhLi}$ & - & -78 & 45 & - & 0 & - \\
\hline 5 & $\mathrm{Et}_{2} \mathrm{O}$ & $1.1 \mathrm{n}-\mathrm{BuLi}$ & $3.0 \mathrm{LiClO}_{4}$ & -78 & 10 & 20 & 17 & $4.7: 1$ \\
\hline 6 & $\mathrm{Et}_{2} \mathrm{O}$ & $1.1 \mathrm{t}-\mathrm{BuLi}$ & $3.0 \mathrm{LiClO}_{4}$ & -78 & 30 & 30 & 27 & $3.1: 1$ \\
\hline 7 & $\mathrm{Et}_{2} \mathrm{O}$ & $1.1 \mathrm{n}-\mathrm{BuLi}$ & $0.86 \mathrm{LDA}$ & -78 & 30 & 60 & 8 & $1: 4.1$ \\
\hline 8 & $\mathrm{Et}_{2} \mathrm{O}$ & $1.1 \mathrm{~s}-\mathrm{BuLi}$ & 0.86 LDA & -78 & 30 & 60 & 13 & $1: 4.3$ \\
\hline 9 & THF & $1.1 \mathrm{n}-\mathrm{BuLi}$ & - & -78 & 20 & - & 73 & $3.1: 1$ \\
\hline 10 & THF & $1.1 \mathrm{~s} \mathrm{BuLi}$ & - & -78 & 30 & - & 89 & $1.4: 1$ \\
\hline 11 & THF & $1.1 \mathrm{t}-\mathrm{BuLi}$ & - & -78 & 20 & - & 72 & $5.1: 1$ \\
\hline 12 & THF & $1.1 \mathrm{PhLi}$ & - & -78 & 45 & - & 37 & $1.2: 1$ \\
\hline 13 & THF & $1.1 \mathrm{n}-\mathrm{BuLi}$ & $3.0 \mathrm{LiClO}_{4}$ & -78 & 10 & 40 & 49 & $2.5: 1$ \\
\hline 14 & THF & $1.1 \mathrm{t}-\mathrm{BuLi}$ & $3.0 \mathrm{LiClO}_{4}$ & -78 & 10 & 40 & 50 & $3.8: 1$ \\
\hline 15 & THF & $1.1 \mathrm{n}-\mathrm{BuLi}$ & 1.1 TMEDA & -78 & 30 & - & 65 & $4.7: 1$ \\
\hline 16 & THF & $1.1 \mathrm{t}-\mathrm{BuLi}$ & 1.1 TMEDA & -78 & 30 & - & 54 & $3.7: 1$ \\
\hline 17 & THF & 1.1 LDA & - & 0 & 30 & - & 0 & - \\
\hline 18 & THF & $1.1 \mathrm{n}-\mathrm{BuLi}$ & 0.86 LDA & -78 & 30 & 150 & 78 & $1: 3.5$ \\
\hline 19 & THF & $1.1 \mathrm{~s}-\mathrm{BuLi}$ & 0.86 LDA & -78 & 60 & 120 & 79 & $1: 4.8$ \\
\hline 20 & THF & $1.1 \mathrm{~s}-\mathrm{BuLi}$ & $1.72 \mathrm{LDA}$ & -78 & 45 & 120 & 94 & $1.1: 1$ \\
\hline 21 & THF & $1.1 \mathrm{t}-\mathrm{BuLi}$ & 0.86 LDA & -78 & 60 & 120 & 67 & $1: 8.8$ \\
\hline 22 & THF & $1.1 \mathrm{t}-\mathrm{BuLi}$ & $1.72 \mathrm{LDA}$ & -78 & 40 & 120 & 58 & $1: 9.2$ \\
\hline 23 & THF & $1.1 \mathrm{~s}-\mathrm{BuLi}$ & $0.86 \mathrm{LDA}$ & -78 & 45 & 120 & 86 & $1: 1.1$ \\
\hline 24 & THF & $1.1 \mathrm{t}-\mathrm{BuLi}$ & $1.15 \mathrm{LDA}$ & -78 & 45 & 120 & 68 & $1: 9.9$ \\
\hline 25 & $\mathrm{THF}$ & $1.1 \mathrm{t}-\mathrm{BuLi}$ & 1.15 LDA & -78 & 15 & 120 & 56 & $1: 10.0$ \\
\hline 26 & THF & $1.1 \mathrm{t}-\mathrm{BuLi}$ & 1.15 LDA & -78 & $10^{*}$ & - & 37 & $1: 7.5$ \\
\hline 27 & THF & $1.1 \mathrm{t}-\mathrm{BuLi}$ & 1.15 LDA & -78 & 45 & 15 & 83 & $1: 6.0$ \\
\hline 28 & THF & $1.1 \mathrm{t}-\mathrm{BuLi}$ & 1.15 LDA & -78 & 60 & 330 & 52 & $1: 7.1$ \\
\hline 29 & THF & $2.1 \mathrm{t}-\mathrm{BuLi}$ & 2.1 LDA & -78 & 60 & 120 & 93 & $1.7: 1$ \\
\hline 30 & $\mathrm{THF}$ & 1.5 t-BuLi & $1.05 \mathrm{LDA}$ & -78 & 45 & 120 & 92 & $2.5: 1$ \\
\hline 31 & THF & $1.00 \mathrm{t}-\mathrm{BuLi}$ & 1.05 LDA & -78 & 45 & 120 & 76 & $1: 9.4$ \\
\hline 32 & THF & 1.00 t-BuLi & 1.50 LDA & -78 & 50 & 30 & 79 & $1: 12.4$ \\
\hline 33 & $\mathrm{THF}$ & $1.1 \mathrm{t}-\mathrm{BuLi}$ & 1.1 LHMDS & -78 & 30 & 120 & 83 & $4.4: 1$ \\
\hline 34 & THF & $1.1 \mathrm{t}-\mathrm{BuLi}$ & 2.2 LHMDS & -78 & 30 & 120 & 51 & $2.7: 1$ \\
\hline 35 & THF & 1.1 LTMP & - & -78 & 45 & 120 & 44 & $1: 5.7$ \\
\hline 36 & THF & $1.1 \mathrm{t}-\mathrm{BuLi}$ & 1.1 LTMP & -78 & 45 & 120 & 95 & $1: 1.6$ \\
\hline 37 & THF & 1.1 $\mathrm{LiNCy}_{2}$ & - & -78 & 30 & - & 0 & - \\
\hline 38 & THF & $1.1 \mathrm{t}-\mathrm{BuLi}$ & 1.1 $\mathrm{LiNCy}_{2}$ & -78 & 45 & 120 & 26 & $1: 4.1$ \\
\hline 39 & THF & $1.1 \mathrm{n}-\mathrm{BuLi}$ & 1.1 $\mathrm{LiNCy}_{2}$ & -78 & 45 & 120 & 42 & $1: 6.2$ \\
\hline 40 & THF & 1.1 $\mathrm{LiNEt}_{2}$ & - & -78 & 30 & - & 0 & - \\
\hline 41 & THF & $1.05 \mathrm{n}-\mathrm{BuLi}$ & 1.1 $\mathrm{LiNEt}_{2}$ & -78 & 45 & 120 & 68 & $1: 5.6$ \\
\hline 42 & THF & $1.05 \mathrm{t}-\mathrm{BuLi}$ & 1.1 $\mathrm{LiNEt}_{2}$ & -78 & 45 & 120 & 55 & $1: 5.2$ \\
\hline 43 & $\mathrm{THF}$ & $2.2 \mathrm{n}-\mathrm{BuLi}$ & 2.2 sparteine & -78 & 15 & 120 & 24 & $1.7: 1$ \\
\hline 44 & $\mathrm{THF}$ & $1.1 \mathrm{n}-\mathrm{BuLi}$ & 1.1 sparteine & -78 & 15 & 120 & 7 & $1.7: 1$ \\
\hline
\end{tabular}

* LDA and $\mathrm{t}-\mathrm{BuLi}$ were premixed and $\mathbf{1 1}$ was added to the mixed aggregate 